\title{
Design of a Novel Flat-plate Photobioreactor System for Green Algal Hydrogen Production
}

B. Tamburic, F.W. Zemichael, G.C. Maitland, K. Hellgardt

This document appeared in

Detlef Stolten, Thomas Grube (Eds.):

18th World Hydrogen Energy Conference 2010 - WHEC 2010

Parallel Sessions Book 2: Hydrogen Production Technologies - Part 1

Proceedings of the WHEC, May 16.-21. 2010, Essen

Schriften des Forschungszentrums Jülich / Energy \& Environment, Vol. 78-2

Institute of Energy Research - Fuel Cells (IEF-3)

Forschungszentrum Jülich $\mathrm{GmbH}$, Zentralbibliothek, Verlag, 2010

ISBN: 978-3-89336-652-1 


\title{
Design of a Novel Flat-plate Photobioreactor System for Green Algal Hydrogen Production
}

\author{
B. Tamburic, F.W. Zemichael, G.C. Maitland, K. Hellgardt, Imperial College \\ London, UK
}

\begin{abstract}
Some unicellular green algae have the ability to photosynthetically produce molecular hydrogen using sunlight and water. This renewable, carbon-neutral process has the additional benefit of sequestering carbon dioxide during the algal growth phase. The main costs associated with this process result from building and operating a photobioreactor system. The challenge is to design an innovative and cost effective photobioreactor that meets the requirements of algal growth and sustainable hydrogen production. We document the details of a novel 1 litre vertical flat-plate photobioreactor that has been designed to accommodate green algal hydrogen production at the laboratory scale. Coherent, nonheating illumination is provided by a panel of cool white LEDs. The reactor body consists of two compartments constructed from transparent Perspex sheets. The primary compartment holds the algal culture, which is agitated by means of a recirculating gas flow. A secondary compartment is filled with water and used to control the temperature and wavelength of the system. The reactor is fitted with instruments that monitor the $\mathrm{pH}, \mathrm{pO}_{2}$, temperature and optical density of the culture. A membrane-inlet mass spectrometry system has been developed for hydrogen collection and in situ monitoring. The reactor is fully autoclaveable and the possibility of hydrogen leaks has been minimised. The modular nature of the reactor allows efficient cleaning and maintenance.
\end{abstract}

\section{Introduction}

\subsection{Green algal hydrogen production}

Molecular hydrogen $\left(\mathrm{H}_{2}\right)$ is a fuel that has the potential to provide the clean energy required for transport, heating and electricity. The aim of our Solar Hydrogen Project is to produce $\mathrm{H}_{2}$ by a carbon-neutral process that uses unlimited natural resources - sunlight and water. The green alga C.reinhardtii has the ability to photosynthetically produce $\mathrm{H}_{2}$ under anaerobic conditions [1]. Initially, photons are absorbed within the chloroplast of C.reinhardtii. This solar energy facilitates the photochemical oxidation of water into protons and molecular oxygen $\left(\mathrm{O}_{2}\right)$ by photosystem II (PSII) proteins. Electrons generated in this process are transferred to the iron-hydrogenase enzyme, which catalyses proton-electron recombination to produce $\mathrm{H}_{2}$. Hydrogenase activity is inhibited in the presence of $\mathrm{O}_{2}$, which implies that this direct biophotolysis process is self-limiting. In order to maintain continuous $\mathrm{H}_{2}$ production, it is necessary to remove $\mathrm{O}_{2}$ as it is being produced. Sulphur deprivation of C.reinhardtii diminishes its ability to repair PSII proteins, thus reducing photosynthetic $\mathrm{O}_{2}$ production below the level of respiratory $\mathrm{O}_{2}$ consumption so that overall, $\mathrm{O}_{2}$ is being used up [2]. Algal metabolism is therefore responsible for creating an anaerobic environment that leads to sustained $\mathrm{H}_{2}$ production. The main barriers to the commercialisation of green algal $\mathrm{H}_{2}$ 
production are the low photochemical conversion efficiencies of the process and the prohibitive photobioreactor (PBR) costs [3].

\subsection{Photobioreactors}

Commercial growth of algal biomass is normally restricted to inexpensive open systems such as natural ponds, circular ponds with a rotating arm for stirring, or raceway ponds [4]. Enclosed PBR systems offer the potential for reproducible cultivation conditions, better biomass and $\mathrm{H}_{2}$ yield, better product quality and the opportunity for flexible technical design [5]. They can enable algal cultivation in arid regions, hence ensuring that algae do not need to compete for land area with food crops, while also opening new economic possibilities in desert countries [6]. The main drawbacks of enclosed PBR systems are their high capital and operating costs. The productivity of a closed PBR is limited by various factors, but most importantly it needs to operate with optimal light requirements, surface-to-volume ratio, agitation time and mass transfer [7]. The types of PBR commonly considered in the literature are the vertical-column reactor, the stirred-tank reactor (often with internal illumination), the tubular reactor, the flat-plate reactor and various immobilised reactors. Flat-plate reactors consist of a rectangular compartment with a depth of $1-5 \mathrm{~cm}$ and a height and width that do not practically exceed 1 metre [8]. Artificially illuminated flat-plate reactors are often vertical, with a light source illuminating the reactor from one side. Outdoor flat-plate reactors are typically tilted at an angle that allows optimal exposure to solar irradiation [4]. All flat-plate reactors are characterised by a high surface-to-volume ratio, which leads to the best photosynthetic efficiencies observed for any PBR [3]. The region immediately adjacent to the illuminated reactor surface is a photic zone where light saturation, and consequently the photoinhibition of algal growth and $\mathrm{H}_{2}$ production processes, repeatedly occurs [9]. In addition, the light energy available to algal cells decreases exponentially away from this photic zone - it has been estimated that for a fully grown culture of C.reinhardtii, effective light penetration is limited to a depth of $0.8 \mathrm{~mm}$ [8]. It is therefore important to minimise these light gradients and control the light-dark cycles of the algal cells by means of an effective agitation system [9]. Flat-plate reactors are subject to relatively low mass transfer rates because the space between panels, known as the light path, is restricted and this reduces the clearance efficiency of the dissolved $\mathrm{O}_{2}$ produced by photosynthesis [10]. Flat-plate reactors provide operational flexibility as they may be run in both batch and continuous modes [5]. Typical limitations are the scaling-up requirements (the need for many compartments and support materials), the difficulty in controlling culture temperature, the possibility of algal wall growth and the incompatibility with certain algal strains [11].

\section{Reactor Design}

\subsection{Design specification}

Our motivation for designing the PBR was to create a simple system that would enable quick measurement of the key parameters in the biohydrogen production process under carefully controlled conditions. As part of the design specification, we made a selection of the parameters that should be controlled and/or measured, used these observations to drive the reactor design, and completed the process by developing the ancillary systems and equipment required to service the reactor. The critical control parameters are temperature, 
light intensity and wavelength, agitation and mixing, nutrient delivery and dilution, and $\mathrm{pH}$ [5]. A continuous, on-line measurement of $\mathrm{H}_{2}$ production is required. Additionally, it is necessary to determine the algal growth rate, the dissolved oxygen concentration (to determine if the culture is anaerobic), and the uptake rates of key nutrients such as sulphate and acetate [11]. Since the availability of light is normally the limiting factor for algal growth and $\mathrm{H}_{2}$ production, we selected the reactor geometry that offers the highest surface-to-volume ratio - the vertical flat-plate reactor. When selecting reactor materials, it was important to consider their strength, durability, spectral properties, toxicity for algal cells, and the diffusion coefficient for $\mathrm{H}_{2}$ [12]. The reactor was designed to be fully autoclaveable to minimise the possibility of contamination, and completely modular to allow easy cleaning and maintenance between experiments [13]. The choice of a minimalistic reactor design and the selection of materials and ancillary systems equipment were made with the intention of keeping the cost of our flat-plate reactor competitive with targets laid out in the literature [2]. The reactor ancillary systems were concerned with illumination, agitation, measurement and datalogging, and user control interface. Illumination was provided by an LED array and the main challenge was to select the correct geometric configuration for optimal light exposure [8]. Diaphragm pumps were used to fulfil the agitation requirements, consisting of turbulent flow, appropriate mixing patterns and low levels of sheer stress on the culture [4]. It was essential to provide instrumentation to measure the $\mathrm{pH}, \mathrm{pO}_{2}, \mathrm{H}_{2}$ production and algal growth rate [13]; commercial probes covered in situ $\mathrm{pH}$ and $\mathrm{pO}_{2}$ measurements, $\mathrm{H}_{2}$ was measured by membrane-inlet mass spectrometry (MIMS) and the algal growth rate was monitored by regularly measuring the optical thickness of the culture using a light meter.

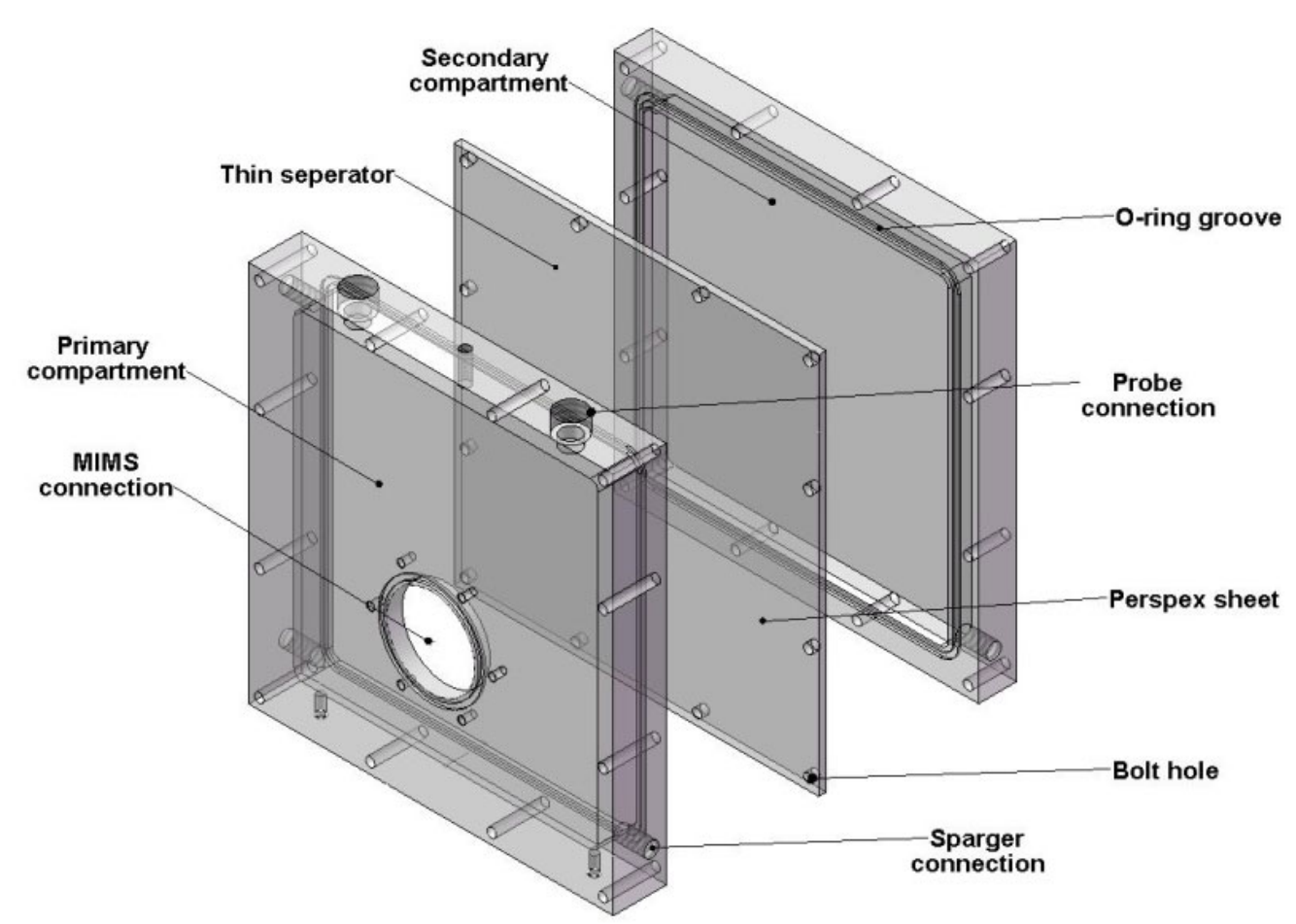

Figure 1: Flat-plate reactor design in Solid Works showing the three-piece Perspex component assembly. 


\subsection{Dual-compartment design}

A novel dual-compartment flat-plate reactor body was designed (Figure 1) and constructed. The primary compartment contains the algal culture, while a secondary compartment is filled with water (or an aqueous solution) and used as a temperature control jacket. Various coloured ions may be dissolved in the secondary compartment to control the intensity and wavelength of illumination reaching the algal culture. The primary compartment also contains a slot for inserting the membrane-inlet mass spectrometry (MIMS) system to be used for $\mathrm{H}_{2}$ measurement. The critical measuring instruments (thermocouple, $\mathrm{pO}_{2}$ and $\mathrm{pH}$ probe) may be attached via the appropriate slots at the top of the primary compartment. The entire structure may be screw-connected onto a firm, black (matt, to minimise surface reflection) polyoxymethylene base, which also holds the LED array with the aid of a support bracket. The final dual-compartment reactor body dimensions were $250 \times 250 \times 70 \mathrm{~mm}$, and the entire construction could fit into a box with dimensions $350 \times 350 \times 22 \mathrm{~mm}$. The primary compartment has a working volume of approximately one litre. The reactor body is made of Perspex (polymethyl methacrylate), a transparent polymer alternative to glass that is relatively inexpensive, autoclaveable and easy to handle and process. Following a number of design iterations, it was decided to construct the reactor body from a thin $(3 \mathrm{~mm})$ Perspex separator sandwiched between two thick $(50 \mathrm{~mm})$ slabs of Perspex. The primary and secondary compartments were machined within these two thick Perspex slabs. The machining was carried out by programming the Solid Works design into the computer-aided machining software Feature Cam. Perspex polish was applied to the machined surfaces to improve their optical transparency. A crucial element of the reactor design was that it should be impermeable to $\mathrm{H}_{2}$. This means that special care had to be taken to ensure a good seal at any location where the culture could potentially be exposed to the surroundings. The Perspex sheets were sealed with an inlaid grooved system of Viton fluoropolymer elastometer o-rings and pressed together using a set of 12 stainless steel bolts, hence also satisfying the requirement for a fully modular design. The agitation system holes were closed by o-ring-sealed stainless steel Swagelok fittings. None of the materials used are known to be toxic to C.reinhardtii.

\subsection{Illumination}

Overall, C.reinhardtii cells have modest light intensity requirements - the growth, sulphur deprivation and $\mathrm{H}_{2}$ production processes need no more than $200 \mu \mathrm{Es}^{-1} \mathrm{~m}^{-2}$ of photosynthetically active radiation (PAR) - about $10 \mathrm{Wm}^{-2}$ [1]. The main objective is to ensure that as many cells as possible have access to uniform illumination [3]. A light emitting diode (LED) array provides illumination that is uniform, coherent, efficient and non-heating, all desirable properties for the flat-plate PBR. Two cool-white LED array panels, approximately 200×200 mm in size, were purchased from LED Wholesalers. Measurements with a light meter (operating on integrating sphere principles) have shown that the spatial light intensity distribution of our LED array, measured directly from a distance of $30 \mathrm{~cm}$ away from the array, is relatively consistent across the flat-plate reactor area, with a light intensity of $58 \pm 3 \mathrm{Wm}^{-2}$. When the reactor is placed between the LED array and light meter, the light intensity drops to $43 \pm 8 \mathrm{Wm}^{-2}$, primarily due to limitations in the transparency of machined Perspex. There is also evidence of additional shading effects radially away from the reactor centre; it is therefore important to make certain all algal cells receive sufficient illumination by 
providing appropriate agitation to the algal culture. It is possible to use a light meter or photodiode in a similar setup to measure the optical thickness of the culture, which is directly proportional to the algal growth rate. Other advantages of LED illumination are that the intensity is readily controllable and that the wavelength shows a good spectral match with C.reinhardtii absorption (Figure 2(a)). C.reinhardtii PSII absorbs in the red region of the visible spectrum, with the main absorption peak at $680 \mathrm{~nm}$ [3]. PSIl absorption is a threshold mechanism, that is to say the algae could use any wavelength lesser-than-or-equal to 680 $\mathrm{nm}$ for photosynthesis, but preferentially use $680 \mathrm{~nm}$, as evidenced by the strong absorption peak at this wavelength. There is also additional carotenoid absorption in the $400-500 \mathrm{~nm}$ spectral range. It is possible to use the coloured aqueous solutions of various ions to modulate the wavelength of illumination incident on the culture (Figure 2(b)). For example, the purple potassium permanganate (aq) could be used to prevent photoinhibition by unnecessary green light. Orange potassium chromate (aq) could be used to switch-off carotenoid absorption, potentially diverting additional photons to PSII, while red cobalt chloride (aq) could be used to combine the above effects.
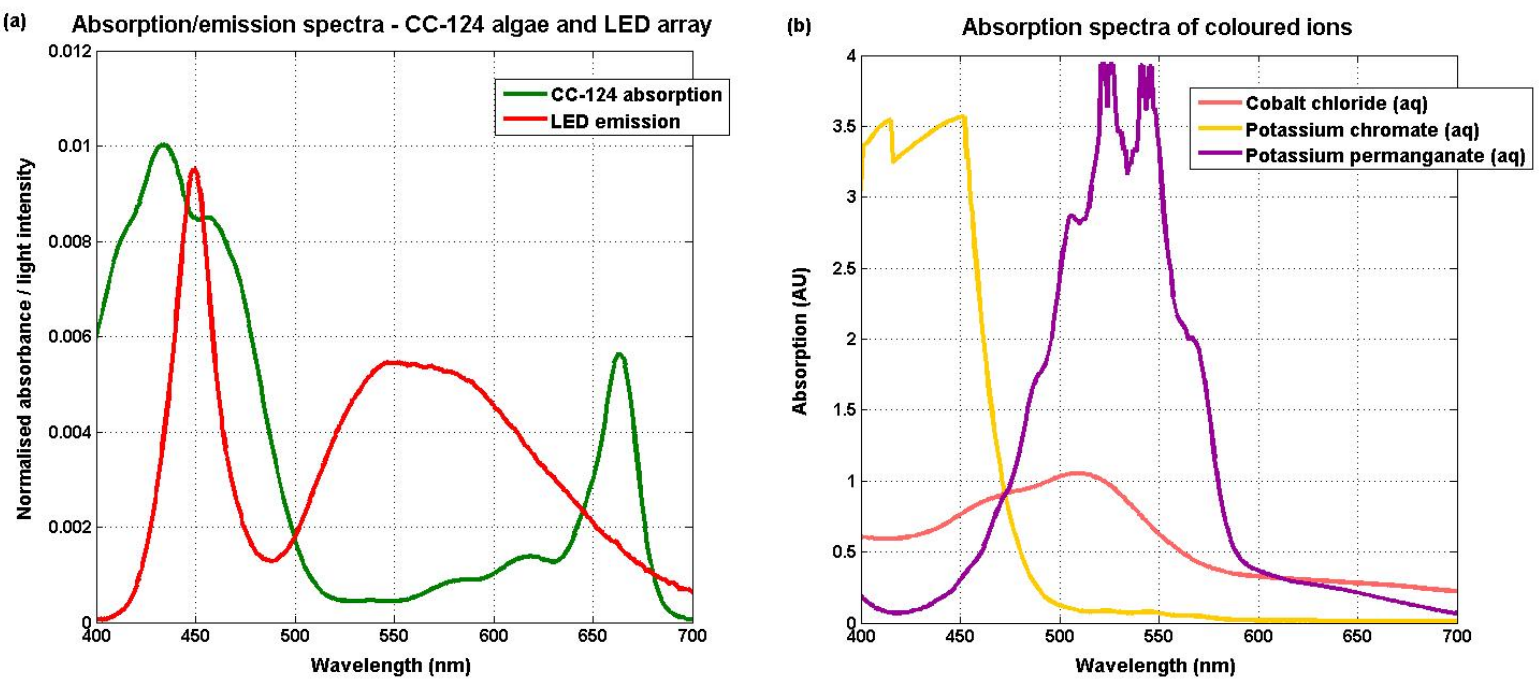

Figure 2: (a) Spectral match between LED emission and C.reinhardtii wild-type CC-124 strain absorption;

(b) absorption spectra of some common coloured aqueous solutions that may be used to modulate LED wavelength.

\section{Reactor Development}

\subsection{Agitation system}

Primary compartment agitation is provided by a turbulent air-lift system emanating from a dual-entry stainless steel sparger at the bottom of the compartment (Figure 1). The gas flow system is enclosed in stainless steel tubes and circulated around the reactor so that no gaseous hydrogen is lost to the environment. Both dissolved and gaseous $\mathrm{H}_{2}$ are collected by a specifically designed MIMS system, which is located on the front end of the primary compartment. Gas is circulated by means of a customised gas diaphragm pump from KNF 
Neuberger. This pump features a stainless steel pumping head with Swagelok inlet/outlet connections to minimise $\mathrm{H}_{2}$ diffusion leaks in this critical region, as well as step-voltage control of the pumping speed. A temperature flux and mass flow model is being developed in Comsol to optimise parameters such as the temperature of the secondary compartment and the flow rates through both compartments. During the algal growth phase, the primary compartment could be agitated by sparging a mixture of carbon dioxide and air, thus providing an additional carbon source for photomixotrophic algal growth. An inert gas such as argon would be used during the anaerobic $\mathrm{H}_{2}$ production phase. Secondary compartment water recirculation is provided by a standard KNF Neuberger liquid diaphragm pump.

\subsection{Membrane-inlet mass spectrometry (MIMS) system}

Various methods have been used to monitor the gaseous species $\left(\mathrm{O}_{2}, \mathrm{CO}_{2}\right.$ and $\left.\mathrm{H}_{2}\right)$ involved during photobiological $\mathrm{H}_{2}$ production. The conventional method has been to use in situ Clarktype electrode measurements and/or analysis of gas samples by gas chromatography. MIMS is another analytical technique used to measure in situ concentrations of dissolved gases or volatile organic compounds in aqueous solutions. The membrane system is the only interface between a liquid sample at atmospheric pressure and the vacuum chamber of a mass spectrometer. The membrane is made from different polymeric materials that allow gases to diffuse simultaneously or selectively depending on the investigation [11]. The technique allows direct comparison of the evolved gases and provides continuous monitoring of process conditions, i.e. light intensity, temperature, $\mathrm{pH}$ and system disturbances. Our flatplate reactor utilises the Aston Analytical Ultra-Trace MIMS System. The mass spectrometer is a compact quadrupole Pfeiffer Vacuum Prisma, running Windows Quadstar 422 software. Gases dissolved in the liquid phase diffuse through a thin polydimethylsiloxane membrane affixed on a perforated disc holder. Inert gas introduced to the disc holder cavity directly carries diffused gaseous products, via a heatable capillary, into the mass spectrometer. To avoid any damage to the vacuum system, a moisture trap is fixed before the inlet of the mass spectrometer. Apart from the direct measurement of gaseous products kinetics, the MIMS system offers the additional benefits of potential $\mathrm{H}_{2}$ separation and collection, as well as the reduction of possible $\mathrm{H}_{2}$ induced inhibition of the biophotolytic $\mathrm{H}_{2}$ production process [14].

\section{Conclusion}

We have successfully designed and constructed a novel dual-compartment flat-plate vertical 1 litre photobioreactor to facilitate biophotolytic $\mathrm{H}_{2}$ production. The primary compartment holds the algal culture and contains the key measuring instruments, including the membraneinlet mass spectrometer system developed for in situ $\mathrm{H}_{2}$ detection and extraction. The secondary compartment is used to control the system temperature and wavelength. The reactor enables quick measurements of the key parameters in the biohydrogen production process under carefully controlled conditions. Preliminary growth and $\mathrm{H}_{2}$ production experiments are now underway in the reactor. 


\section{Acknowledgements}

We would like to thank Paul Crudge, whose technical knowledge was instrumental in the flatplate reactor design and construction process. The Solar Hydrogen project is funded by the UK Engineering and Physical Sciences Research Council (EPSRC).

\section{References}

[1] Ghirardi ML, Zhang L, Lee JW, Flynn T, Seibert M, Greenbaum E, Melis A. Microalgae: a green source of renewable $\mathrm{H}_{2}$. Trends in Biotechnology 2000; 18(12):506-511.

[2] Melis A. Green algal hydrogen production: progress, challenges and prospects. International Journal of Hydrogen Energy 2002; 27(11-12):1217-1228.

[3] Akkerman I, Janssen M, Rocha J, Wijffels RH. Photobiological hydrogen production: photochemical efficiency and bioreactor design. International Journal of Hydrogen Energy 2002; 27(11-12):1195-1208.

[4] Carvalho AP, Meireles LA, Malcata FX. Microalgal reactors: a review of enclosed system design and performances. Biotechnology Progress 2006; 22(6):1490-1506.

[5] Ugwu CU, Aoyagi $\mathrm{H}$, Uchiyama $\mathrm{H}$. Photobioreactors for mass cultivation of algae. Bioresource Technology 2008; 99(10):4021-4028.

[6] Borowitzka MA. Commercial production of microalgae: ponds, tanks, tubes and fermenters. Journal of Biotechnology 1999; 70(1-3):313-321.

[7] Tsygankov AA. Laboratory scale photobioreactors. Applied Biochemistry and Microbiology 2001; 37(4):333-341.

[8] Janssen M, Tramper J, Mur LR, Wijffels RH. Enclosed outdoor photobioreactors: light regime, photosynthetic efficiency, scale-up and future prospects. Biotechnology and Bioengineering 2003; 81(2):193-210.

[9] Hankamer B, Lehr F, Rupprecht J, Mussgnug JH, Posten C, Kruse O. Photosynthetic biomass and $\mathrm{H}_{2}$ production by green algae: from bioengineering to bioreactor scale-up. Physiologia Plantarium 2007; 131(1):10-21.

[10] Sierra E, Acien FG, Fernandez JM, Garcia JL, Gonzales C, Molina E. Characterization of a flat plate photobioreactor for the production of microalgae. Chemical Engineering Journal 2008; 138(1-3):136-147.

[11] Cournac L, Mus F, Bernard L, Guedeney G, Vignais P, Peltier G. Limiting steps of hydrogen production in Chlamydomonas reinhardtii and Synechocystis PCC 6803 as analysed by light-induced gas exchange transients. International Journal of Hydrogen Energy 2002; 27(11-12):1229-1237.

[12] Skjanes $K$, Knutsen $G$, Kallqvist $T$, Lindblad $P$. $H_{2}$ production from marine and freshwater species of green algae during sulphur deprivation and considerations for bioreactor design. International Journal of Hydrogen Energy 2008; 33(2):511-521.

[13] Nedbal L, Trtilek M, Cerveny J, Komarek O, Pakrasi HB. A photobioreactor system for precision cultivation of photoautotrophic microorganisms and high-content analysis of suspension dynamics. Biotechnology and Bioengineering 2008; 100(5):902-910.

[14] Hemschemeier A, Melis A, Happe T. Analytical approaches to photobiological hydrogen production in green algae. Photosynthesis Research 2009; 102(2-3):523540 . 